\title{
Sleep in children improves memory performance on declarative but not procedural tasks
}

\author{
Ines Wilhelm, Susanne Diekelmann, and Jan Born ${ }^{1}$ \\ Department of Neuroendocrinology, University of Lübeck, Lübeck 23538, Germany
}

\begin{abstract}
Sleep supports the consolidation of memory in adults. Childhood is a period hallmarked by huge demands of brain plasticity as well as great amounts of efficient sleep. Whether sleep supports memory consolidation in children as in adults is unclear. We compared effects of nocturnal sleep (versus daytime wakefulness) on consolidation of declarative (word-pair associates, two-dimensional [2D] object location), and procedural memories (finger sequence tapping) in 15 children (6-8 yr) and 15 adults. Beneficial effects of sleep on retention of declarative memories were comparable in children and adults. However, opposite to adults, children showed smaller improvement in finger-tapping skill across retention sleep than wakefulness, indicating that sleep-dependent procedural memory consolidation depends on developmental stage.
\end{abstract}

Compelling evidence has been accumulated that sleep supports the consolidation of newly acquired memories in adults (Maquet 2001; Stickgold 2005; Born et al. 2006). Declarative memories benefit particularly from slow wave sleep (SWS), whereas procedural memories benefit particularly from REM sleep (Plihal and Born 1997; Peigneux et al. 2004; Marshall and Born 2007), aside from distinct contributions of non-REM sleep stage 2 to memory consolidation (Gais et al. 2002; Fogel and Smith 2006; Peters et al. 2007). Childhood, compared with adulthood, is characterized not only by distinctly greater amounts of sleep and SWS (Anders et al. 1995; Ohayon et al. 2004) but also by a tremendous extent of brain and behavioral plasticity, determining the child's capability to rapidly acquire huge amounts of facts and to effectively shape skills in response to environmental challenges ( $\mathrm{Li}$ et al. 2006; Brehmer et al. 2007). However, the role developmental sleep plays for consolidating memory has only been scarcely examined. Restriction of sleep in schoolchildren was shown to be associated to impairments in different cognitive functions (Carskadon et al. 1981; Randazzo et al. 1998; Steenari et al. 2003). Animal studies provided considerable evidence that developmental sleep, like sleep in adults, is crucially involved in brain plasticity (for review, see Dang-Vu et al. 2006). However, in a recent human study (Fischer et al. 2007), children aged 7-11 yr, in contrast to adults, showed impaired rather than improved implicit sequence knowledge in a procedural serial reaction-time task when training was followed by periods of sleep, pointing toward differential dynamics of sleep-dependent consolidation of procedural memories during development.

In the present study, we dissociated effects of post-learning sleep on procedural and declarative types of memories in 15 healthy children (age 6-8 yr, mean \pm SEM: $7.5 \pm 0.16 \mathrm{yr} ; 9$ females, 6 males) and 15 healthy adults $(26.5 \pm 1.3 \mathrm{yr} ; 13$ females, 2 males). The study was approved by the local ethics committee, and informed consent was obtained by participants and the children's parents. All subjects had normal sleep and were adapted to standard polysomnographic recordings (obtained by a portable amplifier, SOMNOscreen EEG 10-20, SOMNOmedics) during a night preceding the actual experiments. Each subject participated in two conditions, a "sleep" and a "wake" condition, which were conducted at the subject's home. The subject's two sessions

\footnotetext{
1Corresponding author.

E-mail born@kfg.mu-luebeck.de; fax 49-4515003640.

Article is online at http://www.learnmem.org/cgi/doi/10.1101/lm.803708.

Freely available online through the Learning \& Memory Open Access option.
}

were separated by an interval of at least $1 \mathrm{wk}$, and the order of conditions was balanced across subjects. In the sleep condition, learning started at 8:00 p.m. for the children and at 10:00 p.m. for the adults after subjects had been prepared for polysomnographic recordings. Subjects went to bed and lights were turned off at the habitual time for children (between 7:30 and 9:30 p.m.) and adults (between 10:00 p.m. and 12:00 a.m.). The next morning subjects were awakened at their usual time. Retrieval testing took place $\sim 60$ min later, thus avoiding any substantial effects of sleep inertia on performance (Tassi et al. 2006; Wertz et al. 2006). The interval between learning and retrieval testing averaged 11 $\mathrm{h}$. In the wake condition, learning took place in the morning $\sim 60$ min after awakening from nighttime sleep, and retrieval was tested after a retention interval of wakefulness that again lasted $\sim 11 \mathrm{~h}$. During the wake retention interval, subjects followed their daily schedules. The parents kept a continuous record of the children's activities in order to exclude possible disturbing influences by extraordinary stress or interfering cognitive activities. Before learning and retrieval testing, in both conditions, the subjects estimated their subjective tiredness and motivation. Children did so by oral report, and adults filled in a standardized questionnaire.

To assess retention of declarative and procedural memories, three different tasks (each in two parallel versions) were applied. Declarative memory was tested using a word-pair associate learning task and a two-dimensional (2D) object location task. Procedural memory was tested by a finger sequence tapping task. In each session (during both encoding and retest), the $2 \mathrm{D}$ object location task was tested first, followed by finger sequence tapping and the word-pair associate learning task. The word-pair associate learning task required the children to learn a list of 20 wordpairs. For adults the list included 40 word-pairs in order to achieve a roughly comparable task difficulty in both age groups. At learning, the experimenter read out loud all word-pairs of the list ( 1 word-pair/5 sec). Then, she read the first (cue) word of each pair in random order, and the subject had to name the associated word (with no explicit time limit). This procedure was repeated until the subject reached a criterion of $60 \%$ correct responses. At retrieval testing after the retention interval, the same cued recall procedure was used with each word-pair tested once. The word-pairs were taken mainly from the Handbuch deutschsprachiger Wortnormen (Hager and Hasselhorn 1994) and were of low emotionality and highly concrete and imaginable. They were moderately associated according to examinations in age- 
matched samples of children and adults and, thus, differed for both age groups (e.g., for adults, "arm-bandage;" for children, "arm-blood").

The 2D object location task (adopted from Rasch et al. 2007) resembles the game "concentration" and consists of 15 card-pairs showing colored pictures of different animals and everyday objects. A cued recall procedure was repeated until the criterion was reached, which was set to $40 \%$ and $60 \%$ correct responses in children and adults, respectively, to achieve comparable task difficulty. At retrieval testing after the retention interval, all cardpairs were tested once using the same cued recall procedure.

The finger sequence tapping task was adopted from Walker et al. (2003a) with slight modifications to adjust it for use in young children. It requires the subject to press repeatedly one of two five-element sequences (4-1-3-2-4 and 2-3-1-4-2) on a keyboard with the fingers of his nondominant hand. The subjects were instructed to repeatedly type the sequence as fast and as accurately as possible. To keep working memory demands (which were expected to affect more strongly performance in children than in adults) at an absolute minimum, four horizontally arranged boxes, corresponding to the keys, were displayed on a screen in front of the subject, and a white star successively appeared in the box cuing the next key to be pressed. At learning, subjects performed on twelve 30 -sec trials each interrupted by 30 -sec breaks. Retrieval testing included three trials which were performed after subjects had one warm-up trial. (Inclusion of this warm-up trial in the analyses did not change results.) To control for nonspecific changes in motor performance, at retrieval testing a new sequence was introduced subsequent to retrieval testing. Performance on three trials of this novel sequence was tested after subjects had one practice trial.

Memory retention on the word-pair associate task and the $2 \mathrm{D}$ object location task was determined by the difference in the number of items recalled at retrieval testing minus the number of recalled items on the criterion trial at learning before the retention interval. On the word-pair associate task, in both children and adults retention of word-pairs across the sleep interval was better than across the wake interval $\left(F_{(1,28)}=17.14 ; P<0.001\right.$, for main effect of "sleep/wake" in a $2 \times 2$ analysis of variance across both groups; $P<0.01$ and $P<0.05$, for separate pairwise comparisons between sleep and wake conditions in children and adults, respectively; Fig. 1A). Also, both children and adults remembered more card locations on the $2 \mathrm{D}$ object location task after the sleep retention interval than the wake retention interval $\left(F_{(1,28)}=10.77 ; P<0.01\right.$, for main effect of "sleep/wake"; $P<0.01$ and $P<0.05$, for pairwise comparisons between sleep and wake conditions in children and adults, respectively; Fig. 1B). Retention rates in terms of the relative difference in performance at retrieval minus performance at learning in both declarative memory tasks were comparable between children and adults ( $P>0.35$, main effect of "age," for both comparisons), and so was the enhancing effect of sleep on retention rates $(P>0.40$, "sleep/ wake" $\times$ "age" interaction, for both comparisons). Performance at learning (i.e., criterion performance and trials to criterion) was closely comparable between sleep and wake conditions in children and adults $(P>0.20$ for all comparisons). The number of trials to reach the criterion did not differ between children and adults $(P>0.05)$, which indicates that difficulty of the tasks was comparable between children and adults. However, in light of the apparent differences in cognitive functioning between the age groups, any comparison regarding task difficulty basically remains tentative.

Finger sequence tapping performance was assessed with regard to accuracy (number of correct key presses per trial) and speed (number of key presses per trial). Since both measures revealed essentially the same results, this report is restricted to the
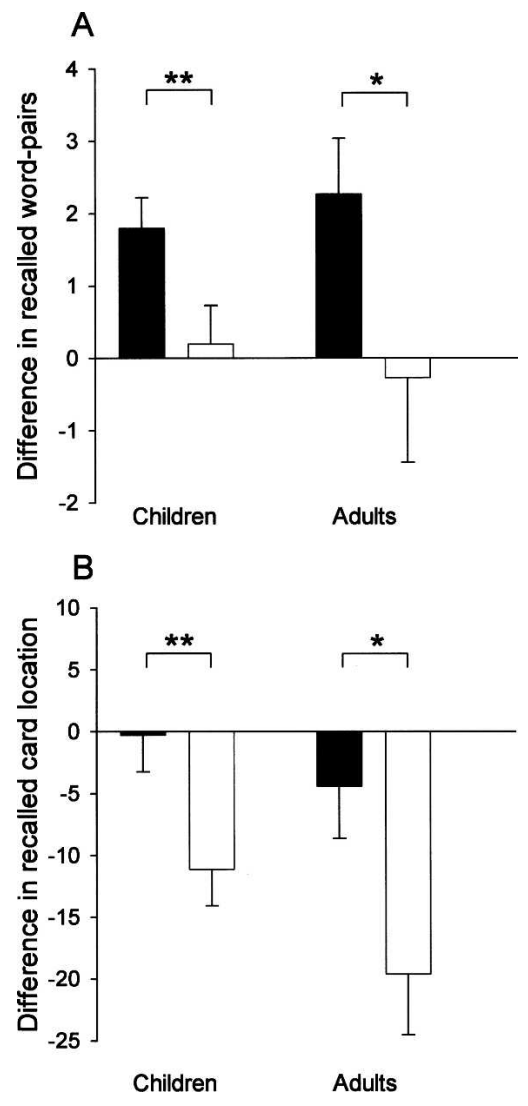

Figure 1. Retention performance on both declarative memory tasks in children and adults. Mean $( \pm$ SEM) retention of word-pairs on the wordpair associate learning task $(A)$ and of card locations on the 2D object location task $(B)$ across intervals of nocturnal sleep (black bars) and daytime wakefulness (white bars) in children (left) and adults (right). Retention performance is indicated by the absolute difference in the number of recalled items (word-pairs and card locations, respectively) at retrieval testing after the retention interval minus the number of items recalled at the criterion trial at learning before the retention interval. ${ }^{*} P<0.05$, $* \star P<0.01$, for pairwise comparisons between sleep and wake conditions within age groups.

accuracy measure. Changes in performance across the retention intervals were determined by the difference in average performance on the three retrieval trials minus performance on the last three trials at learning. Data from one child not completing the task were excluded. Changes in finger sequence tapping performance between learning and retrieval testing were in opposite direction in children and adults $\left(F_{(1,27)}=7.77 ; P<0.01\right.$; for "sleep/wake" $\times$ "age"). In adults, compared with wakefulness, sleep during the retention interval caused the expected gain in motor skill (increase in number of correct key presses per trial with reference to learning; sleep, $21.2 \pm 4.27$; wake, $8.3 \pm 3.68$; $P<0.05)$. In contrast, children showed better performance after the wake retention interval compared to sleep (sleep, $4.2 \pm 1.97$; wake, $8.1 \pm 1.00 ; P<0.05$; Fig. $2 \mathrm{~A}$ ). In children, the absolute measure of performance on the novel sequence introduced at retrieval testing was also better in the wake condition than in the sleep condition $\left(F_{(1,13)}=4.88 ; P<0.05\right)$. (However, differences in performance between the novel and learned sequence did not differ between sleep and wake conditions, $P>0.40$.) In the adults, performance on this novel sequence did not differ between conditions $(P>0.65)$. Analysis of the 12 trials of initial training revealed that, as expected, children were generally slower than adults $\left(F_{(1,27)}=197.84, P<0.001\right.$, main effect of 


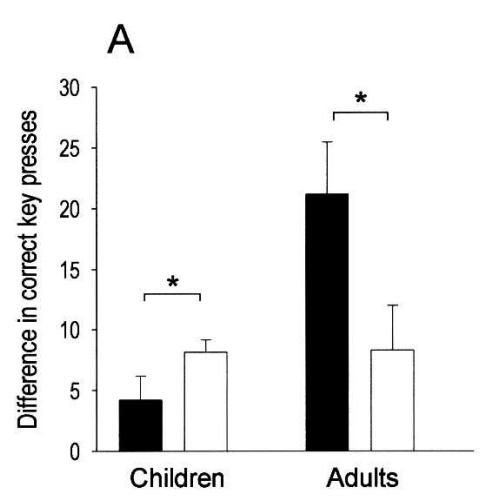

Children

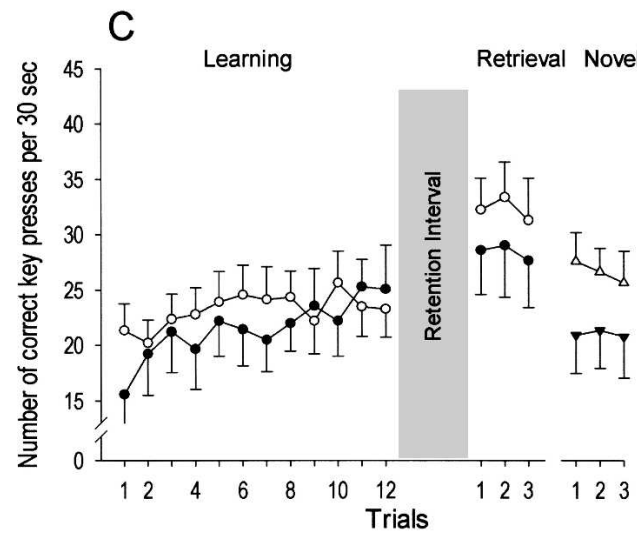

B

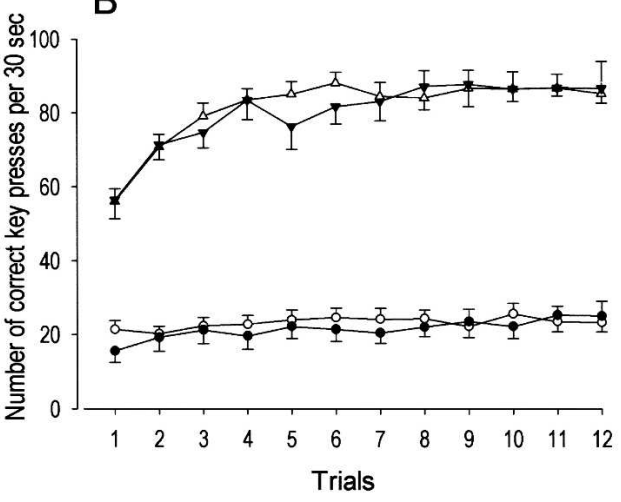

Adults

D

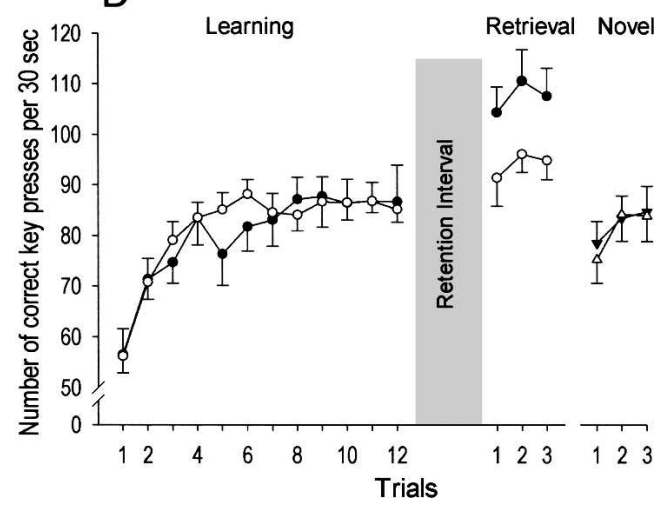

Figure 2. Finger sequence tapping performance in children and adults. Mean ( \pm SEM) values are indicated. $(A)$ Gain in finger tapping skill across retention periods of nocturnal sleep (black bars) and daytime wakefulness (white bars). Gains in skill are determined by the difference in the number of correct key presses per 30-sec trial between performance at the end of learning (trials 10-12) and at the three trials of retrieval testing (note: analysis including the last four learning trials revealed essentially the same results); ${ }^{*} P<0.05$, for pairwise comparisons within age groups. (B) Performance at learning in children (circles) and adults (triangles) before retention intervals of nocturnal sleep (filled symbols) and daytime wakefulness (open symbols). Learning included twelve 30 -sec trials. Note distinctly slower performance in children than adults. $(C, D)$ Summaries of finger sequence tapping performance separately in children and adults for the 12 trials at learning before retention intervals of sleep (filled symbols) and wakefulness (open symbols) and for the three test trials at retrieval thereafter. Following retrieval, performance was tested on a novel sequence that had not been trained at learning. In children, performance on the novel sequence was worse $(P<0.05)$ after the sleep retention interval than after the wake retention interval. Performance in $B-D$ is indicated by the number of correct key presses per 30 -sec trial.

"age;" Fig. 2B). Performance across the training trials clearly improved in both groups $\left(F_{(11,297)}=13.8, P<0.001\right.$, for main effect of "trial" in an ANOVA on all learning trials, $P<0.05$ for separate ANOVA in children and adults), with the children, however, displaying a smaller improvement than adults $\left(F_{(11,297)}=8.08\right.$, $P<0.001$, for "trial" $\times$ "age"). Importantly, training performance did not differ between sleep and wake conditions, neither in children nor in adults $(P>0.15$, for all comparisons including tests at single time points). Based on studies suggesting that delayed gains in skill depend on whether improvement during training had reached saturation (Hauptmann et al. 2005), we analyzed saturation by calculating the difference in improvement as estimated by linear regression beta weights across trials 1-4 versus trials 9-12. Thus, a great difference value indicated performance levels close to saturation in the end of training. As expected, adults achieved higher levels of saturation during learning than children $\left(F_{(1,27)}=8.33, P<0.01\right)$. However, there was no significant correlation of saturation with any measure of retention performance.

Compared with adults, children slept longer $(t=6.13$; $P<0.001)$ and had a lower proportion of lighter non-REM sleep stage $2(t=-2.92 ; P<0.01)$ and a greater proportion of SWS $(t=2.31 ; P<0.05$; Table 1$)$. Children showed a shorter SWS la- tency $(t=-4.02 ; P<0.001)$ and a longer REM sleep latency $(t=7.87 ; P<0.001)$ than adults. None of the sleep parameters was significantly associated with the gain in memory performance for any of the three tasks in both age groups $(r<0.32)$, consonant with the view that time in certain sleep stages per se does not sensitively reflect the processes that mediate memory consolidation (Born et al. 2006). Subjective feelings of tiredness and motivation rated before learning and before retrieval did not indicate any differences between the sleep and the wake condition in adults or children.

Our results in adults replicate previous findings showing that retention of declarative and procedural memories benefit from a period of sleep in comparison with wakefulness after learning (Walker et al. 2003b; Gais and Born 2004; Walker et al. 2005; Ellenbogen et al. 2006; Gais et al. 2006; Robertson and Cohen 2006). By contrast, in the children the effect of sleep on retention depends on the type of memory task: like in adults, retention of declarative memories was distinctly enhanced when sleep followed learning. However, finger sequence tapping improved more over daytime wakefulness than nighttime sleep, speaking for a differential role of sleep for the "offline" consolidation of procedural memories during development.

Our data provide further evidence that the supportive effect 
Table 1. Sleep during nocturnal retention interval

\begin{tabular}{lcc}
\hline & Children & \multicolumn{1}{c}{ Adults } \\
\hline Total sleep time (min) & $567.05 \pm 16.59^{\star *}$ & $413.15 \pm 17.93$ \\
Sleep onset (min) & $17.25 \pm 3.81^{\mathrm{a}}$ & $22.38 \pm 5.76$ \\
SWS latency (min) & $8.65 \pm 0.82^{* *}$ & $18.04 \pm 1.94$ \\
REM latency (min) & $174.60 \pm 5.92^{\star *}$ & $80.96 \pm 9.35$ \\
Sleep stages, time in minutes & & \\
$\quad$ Wake time & $12.55 \pm 8.41$ & $5.54 \pm 1.38$ \\
Stage 1 sleep & $14.80 \pm 3.90$ & $13.54 \pm 2.88$ \\
Stage 2 sleep & $239.75 \pm 17.51$ & $215.54 \pm 11.92$ \\
SWS & $170.60 \pm 22.36^{* *}$ & $82.61 \pm 12.19$ \\
REM sleep & $128.15 \pm 7.06^{* *}$ & $94.69 \pm 9.00$ \\
Sleep stages, time in percent & & \\
$\quad$ & \\
Of total sleep time & & \\
Wake time & $2.35 \pm 1.63$ & $1.33 \pm 0.33$ \\
Stage 1 sleep & $2.69 \pm 0.78$ & $3.23 \pm 0.61$ \\
Stage 2 sleep & $42.31 \pm 2.67^{* *}$ & $52.46 \pm 2.26$ \\
SWS & $29.92 \pm 3.73^{*}$ & $20.01 \pm 2.44$ \\
REM sleep & $22.52 \pm 0.90$ & $22.65 \pm 1.59$ \\
\end{tabular}

Mean ( \pm SEM) total sleep time, time to sleep onset (after lights off), latency of first period of SWS and REM sleep (with reference to sleep onset), and time spent awake, in stage 1 sleep, stage 2 sleep, SWS (i.e., sum of stages 3 and 4), and REM sleep given in minutes and percentage of total sleep time. Asterisks indicate significant difference in children in comparison with adults: ${ }^{*} P<0.05,{ }^{*} P<0.01$

a Sleep onset time in children is biased because they were allowed to follow habitual routines (like listening to a song) after turning off lights.

of sleep on hippocampus-dependent declarative memory consolidation observed in adults is likewise present in young, 6- to 8 -yr-old children. Declarative memory consolidation has been linked to SWS the amount of which was distinctly higher in children than adults (Marshall and Born 2007). Whether this excess SWS enabled an even greater declarative memory benefit in children than adults, cannot be answered because the differences in pre-existing knowledge between both age groups and corresponding differences in the tasks (introduced to adjust task difficulty) precludes any direct quantitative comparison (Bjorklund and Schneider 1996).

Although surprising at a first glance, our finding in children of greater gains in finger motor skill across a wake retention interval than a sleep retention interval agrees with two previous human studies indicating likewise relatively diminished sleepdependent gains in motor sequence learning and language skills (exact recognition of artificial words), respectively (Gomez et al. 2006; Fischer et al. 2007). In combination with equivalent findings in young birds showing deteriorated song performance after sleep (Deregnaucourt et al. 2005), these findings strongly speak for the notion that sleep during development exerts a specific effect on the offline learning of a skill that differs from that in adults and manifests itself in a relatively impaired performance at retrieval, if compared with performance after a wake control interval. However, an immediate lack of overnight gains in skill does not necessarily exclude an improving influence of posttraining sleep on the long term: The birds that showed stronger post-sleep deterioration during development achieved a better final imitation at the end of the 3-mo study epoch (Deregnaucourt et al. 2005).

Unspecific factors like tiredness, motivation, or mood, as well as unfamiliarity with the experimental conditions or sleep inertia, were all carefully controlled by the experimental procedure and thus are unlikely to have confounded memory performance in our subjects. Importantly, if such factors exerted any influence in children, the consolidation of declarative memory should also have been affected. Also, learning performance was closely comparable between morning and evening sessions in both age groups ( $P>0.5$ for all comparisons), and we could con- firm this negative finding in an additional study in 16 children in the same age range for learning on 30 trials of a similar finger tapping task with a longer (12 positions) sequence $(P>0.3$, for all comparisons between morning and evening sessions). Although circadian effects on finger motor performance may occur under specific conditions (Keisler et al. 2007), these findings rule out that such effects substantially confounded finger motor performance at learning or retrieval testing in the present study. However, since we compared effects of sleep that occurred during nighttime with those of daytime wakefulness a confounding influence of circadian rhythm on putative consolidation processes during sleep cannot be entirely excluded, although a substantial bias resulting from this factor seems unlikely in light of numerous previous studies indicating that consolidation processes occurring during nighttime and daytime sleep are basically equivalent (Fischer et al. 2002; Mednick et al. 2003).

Lacking sleep-dependent gains in motor skill in our children might have been due to the lacking saturation of the children's performance during training. Compared with adults' performance, in our children, finger tapping speed was distinctly slower and also did not level off to reach an asymptotic level of automatization toward the end of training. However, in contrast to previous studies in adults (Hauptmann et al. 2005), we did not reveal a significant correlation of overnight performance gains with saturation measures of performance during training in our children. Yet, a lacking linear correlation might not entirely rule out insufficient saturation as a factor contributing to the changed overnight dynamics in motor skill consolidation in children.

Possible interactions between procedural and hippocampusdependent declarative memory systems are also to be considered in this context. There is increasing evidence that formation of procedural memories is not achieved as independently from hippocampal function as originally assumed, which holds also for the finger sequence tapping task (S. Corkin, referenced in Diekelmann and Born 2007). Particularly, at initial stages of skill acquisition and most likely also during consolidation, explicit mechanisms involving hippocampal function interfere with implicit procedural aspects of performance (Jimenez et al. 1996; Willingham and Goedert-Eschmann 1999; Wagner et al. 2004; Forkstam and Petersson 2005; Fischer et al. 2006; Foerde et al. 2006). Compared with adults' performance, in our children, finger tapping speed was distinctly slower, and many of the children tended to speak out loud the respective numbers allocated to the bars to be pressed, indicating that they relied strongly on explicit learning strategies involving hippocampal in addition to prefrontal functions. Competitive interference from hippocampus-dependent memory systems might have been furthered by the fixed task order used in our study, with the word-pair associate task always following the finger tapping task. However, in a recent study in adults (Brown and Robertson 2007), an interpolated declarative task blocked offline improvements across wake intervals only but not across nocturnal sleep. Thus, task order is not likely a factor that can fully account for the lacking overnight gain of skill in our children. Nevertheless, competition from hippocampusdependent mechanisms of memory consolidation during succeeding sleep could be disproportionately enhanced in the children because of their high amounts of SWS which has been revealed to strengthen preferentially hippocampally encoded aspects of task performance (Plihal and Born 1997; Marshall et al. 2006; Backhaus et al. 2007; Marshall and Born 2007). On the other hand, the relatively strong motor skill improvement across the wake interval in our children, consonant with previous reports (Dorfberger et al. 2007), suggests that during development major aspects of procedural memory consolidation per se take place in the waking state. 


\section{Acknowledgments}

We thank Anja Otterbein and Monique Friedrich for technical support and Björn Rasch for fruitful discussion of the data. This study was supported by a grant from the Deutsche Forschungsgemeinschaft SFB 654 "Plasticity and Sleep."

\section{References}

Anders, T.F., Sadeh, A., and Appareddy, V. 1995. Normal sleep in neonates and children. In Principles and practices of sleep medicine in the child (eds. R. Ferber and M. Kryger), pp. 7-18. W.B. Saunders, Philadelphia.

Backhaus, J., Hoeckesfeld, R., Born, J., Hohagen, F., and Junghanns, K. 2007. Immediate as well as delayed post learning sleep but not wakefulness enhances declarative memory consolidation in children. Neurobiol. Learn. Mem. 89: 76-80.

Bjorklund, D.F. and Schneider, W. 1996. The interaction of knowledge, aptitude, and strategies in children's memory performance. Adv. Child Dev. Behav. 26: 59-89.

Born, J., Rasch, B., and Gais, S. 2006. Sleep to remember. Neuroscientist 12: $410-424$.

Brehmer, Y., Li, S.C., Muller, V., von Oertzen, T., and Lindenberger, U. 2007. Memory plasticity across the life span: Uncovering children's latent potential. Dev. Psychol. 43: 465-478.

Brown, R.M. and Robertson, E.M. 2007. Off-line processing: Reciprocal interactions between declarative and procedural memories. $J$. Neurosci. 27: 10468-10475.

Carskadon, M.A., Harvey, K., and Dement, W.C. 1981. Sleep loss in young adolescents. Sleep 4: 299-312.

Dang-Vu, T.T., Desseilles, M., Peigneux, P., and Maquet, P. 2006. A role for sleep in brain plasticity. Pediatr. Rehabil. 9: 98-118.

Deregnaucourt, S., Mitra, P.P., Feher, O., Pytte, C., and Tchernichovski, O. 2005. How sleep affects the developmental learning of bird song. Nature 433: 710-716.

Diekelmann, S. and Born, J. 2007. One memory, two ways to consolidate? Nat. Neurosci. 10: 1085-1086.

Dorfberger, S., Adi-Japha, E., and Karni, A. 2007. Reduced susceptibility to interference in the consolidation of motor memory before adolescence. PLOS ONE 2: e240. doi: 10.1371/journal.pone.0000240.

Ellenbogen, J.M., Hulbert, J.C., Stickgold, R., Dinges, D.F., and Thompson-Schill, S.L. 2006. Interfering with theories of sleep and memory: Sleep, declarative memory, and associative interference. Curr. Biol. 16: 1290-1294.

Fischer, S., Drosopoulos, S., Tsen, J., and Born, J. 2006. Implicit learning-Explicit knowing: A role for sleep in memory system interaction. J. Cogn. Neurosci. 18: 311-319.

Fischer, S., Hallschmid, M., Elsner, A.L., and Born, J. 2002. Sleep forms memory for finger skills. Proc. Natl. Acad. Sci. 99: 11987-11991.

Fischer, S., Wilhelm, I., and Born, J. 2007. Developmental differences in sleep's role for implicit off-line learning: Comparing children with adults. J. Cogn. Neurosci. 19: 214-227.

Foerde, K., Knowlton, B.J., and Poldrack, R.A. 2006. Modulation of competing memory systems by distraction. Proc. Natl. Acad. Sci. 103: $11778-11783$.

Fogel, S.M. and Smith, C.T. 2006. Learning-dependent changes in sleep spindles and stage 2 sleep. J. Sleep Res. 15: 250-255.

Forkstam, C. and Petersson, K.M. 2005. Towards an explicit account of implicit learning. Curr. Opin. Neurol. 18: 435-441.

Gais, S. and Born, J. 2004. Declarative memory consolidation: Mechanisms acting during human sleep. Learn. Mem. 11: 679-685.

Gais, S., Molle, M., Helms, K., and Born, J. 2002. Learning-dependent increases in sleep spindle density. J. Neurosci. 22: 6830-6834.

Gais, S., Lucas, B., and Born, J. 2006. Sleep after learning aids memory recall. Learn. Mem. 13: 259-262.

Gomez, R.L., Bootzin, R.R., and Nadel, L. 2006. Naps promote abstraction in language-learning infants. Psychol. Sci. 17: 670-674.

Hager, W. and Hasselhorn, M. 1994. Handbuch deutschsprachiger Wortnormen. Hogrefe, Göttingen.

Hauptmann, B., Reinhart, E., Brandt, S.A., and Karni, A. 2005. The predictive value of the leveling off of within session performance for procedural memory consolidation. Brain Res. Cogn. Brain Res. 24: $181-189$.

Jimenez, L., Méndez, C., and Cleeremans, A. 1996. Comparing direct and indirect measures of sequence learning. J. Exp. Psychol. Learn. Mem. Cogn. 22: 948-969.

Keisler, A., Ashe, J., and Willingham, D.T. 2007. Time of day accounts for overnight improvement in sequence learning. Learn. Mem. 14: 669-672.

Li, S.C., Brehmer, Y., Shing, Y.L., Werkle-Bergner, M., and Lindenberger, U. 2006. Neuromodulation of associative and organizational plasticity across the life span: Empirical evidence and neurocomputational modeling. Neurosci. Biobehav. Rev. 30: 775-790.

Maquet, P. 2001. The role of sleep in learning and memory. Science 294: $1048-1052$.

Marshall, L. and Born, J. 2007. The contribution of sleep to hippocampus-dependent memory consolidation. Trends Cogn. Sci. 11: $442-450$.

Marshall, L., Helgadottir, H., Molle, M., and Born, J. 2006. Boosting slow oscillations during sleep potentiates memory. Nature 444: 610-613.

Mednick, S., Nakayama, K., and Stickgold, R. 2003. Sleep-dependent learning: A nap is as good as a night. Nat. Neurosci. 6: 697-698.

Ohayon, M.M., Carskadon, M.A., Guilleminault, C., and Vitiello, M.V. 2004. Meta-analysis of quantitative sleep parameters from childhood to old age in healthy individuals: Developing normative sleep values across the human lifespan. Sleep 27: 1255-1273.

Peigneux, P., Laureys, S., Fuchs, S., Collette, F., Perrin, F., Reggers, J., Phillips, C., Degueldre, C., Del Fiore, G., Aerts, J., et al. 2004. Are spatial memories strengthened in the human hippocampus during slow wave sleep? Neuron 44: 535-545.

Peters, K.R., Smith, V., and Smith, C.T. 2007. Changes in sleep architecture following motor learning depend on initial skill level. $J$. Cogn. Neurosci. 19: 817-829.

Plihal, W. and Born, J. 1997. Effects of early and late nocturnal sleep on declarative and procedural memory. J. Cogn. Neurosci. 9: 534-547.

Randazzo, A.C., Muehlbach, M.J., Schweitzer, P.K., and Walsh, J.K. 1998. Cognitive function following acute sleep restriction in children ages 10-14. Sleep 21: 861-868.

Rasch, B., Buchel, C., Gais, S., and Born, J. 2007. Odor cues during slow-wave sleep prompt declarative memory consolidation. Science 315: $1426-1429$.

Robertson, E.M. and Cohen, D.A. 2006. Understanding consolidation through the architecture of memories. Neuroscientist 12: 261-271.

Steenari, M.R., Vuontela, V., Paavonen, E.J., Carlson, S., Fjallberg, M. and Aronen, E. 2003. Working memory and sleep in 6- to 13-year-old schoolchildren. J. Am. Acad. Child Adolesc. Psychiatry 42: 85-92.

Stickgold, R. 2005. Sleep-dependent memory consolidation. Nature 437: $1272-1278$.

Tassi, P., Bonnefond, A., Engasser, O., Hoeft, A., Eschenlauer, R., and Muzet, A. 2006. EEG spectral power and cognitive performance during sleep inertia: The effect of normal sleep duration and partial sleep deprivation. Physiol. Behav. 87: 177-184.

Wagner, U., Gais, S., Haider, H., Verleger, R., and Born, J. 2004. Sleep inspires insight. Nature 427: 352-355.

Walker, M.P., Brakefield, T., Hobson, J.A., and Stickgold, R. 2003a. Dissociable stages of human memory consolidation and reconsolidation. Nature 425: 616-620.

Walker, M.P., Brakefield, T., Seidman, J., Morgan, A., Hobson, J.A., and Stickgold, R. 2003b. Sleep and the time course of motor skill learning. Learn. Mem. 10: 275-284.

Walker, M.P., Stickgold, R., Alsop, D., Gaab, N., and Schlaug, G. 2005. Sleep-dependent motor memory plasticity in the human brain. Neuroscience 133: 911-917.

Wertz, A.T., Ronda, J.M., Czeisler, C.A., and Wright Jr., K.P. 2006. Effects of sleep inertia on cognition. JAMA 295: 163-164.

Willingham, D. and Goedert-Eschmann, K. 1999. The relation between explicit and implicit learning: Evidence for parallel learning. Psychol. Sci. 10: $531-534$.

Received October 11, 2007; accepted in revised form February 15, 2008. 


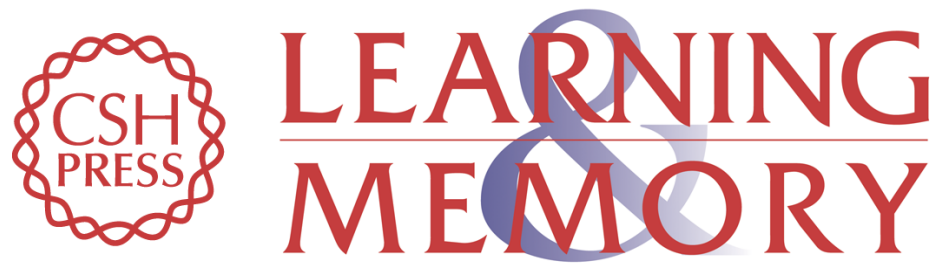

\section{Sleep in children improves memory performance on declarative but not procedural tasks}

Ines Wilhelm, Susanne Diekelmann and Jan Born

Learn. Mem. 2008, 15:

Access the most recent version at doi:10.1101//m.803708

References This article cites 45 articles, 10 of which can be accessed free at: http://learnmem.cshlp.org/content/15/5/373.full.html\#ref-list-1

License Freely available online through the Learning \& Memory Open Access option.

Email Alerting Receive free email alerts when new articles cite this article - sign up in the box at the Service top right corner of the article or click here. 